\title{
BDNF levels in adolescent patients with anorexia nervosa increase continuously to supranormal levels 2.5 years after first hospitalization
}

\author{
Britta Borsdorf, MD; Brigitte Dahmen, MD; Katharina Buehren, MD; Astrid Dempfle, PhD; \\ Karin Egberts, MD; Stefan Ehrlich, MD, PhD; Christian Fleischhaker, MD; \\ Kerstin Konrad, PhD; Reinhild Schwarte, MD; Nina Timmesfeld, PhD; \\ Christoph Wewetzer, MD; Ronald Biemann, MD; Wolfgang Scharke, PhD; \\ Beate Herpertz-Dahlmann, MD; Jochen Seitz, MD
}

\begin{abstract}
Background: Brain-derived neurotrophic factor (BDNF) influences brain plasticity and feeding behaviour, and it has been linked to anorexia nervosa in numerous studies. Findings in mostly adult patients point to reduced serum BDNF levels in the acute stage of anorexia nervosa and rising levels with weight recovery. However, it is unclear whether this increase leads to normalization or supranormal levels, a difference that is potentially important for the etiology of anorexia nervosa and relapse. Methods: We measured serum BDNF at admission $(n=149)$, discharge $(n=130)$, 1-year follow-up $(n=116)$ and 2.5-year follow-up $(n=76)$ in adolescent female patients with anorexia nervosa hospitalized for the first time, and in healthy controls $(n=79)$. We analyzed associations with body mass index, eating disorder psychopathology and comorbidities. Results: Serum BDNF was only nominally lower at admission in patients with anorexia nervosa compared to healthy controls, but it increased continuously and reached supranormal levels at 2.5-year follow-up. BDNF was inversely associated with eating disorder psychopathology at discharge and positively associated with previous weight gain at 1-year follow-up. Limitations: We compensated for attrition and batch effects using statistical measures. Conclusion: In this largest longitudinal study to date, we found only nonsignificant reductions in BDNF in the acute stage of anorexia nervosa, possibly because of a shorter illness duration in adolescent patients. Supranormal levels of BDNF at 2.5-year follow-up could represent a pre-existing trait or a consequence of the illness. Because of the anorexigenic effect of BDNF, it might play an important predisposing role for relapse and should be explored further in studies that test causality.
\end{abstract}

\section{Introduction}

Patients with anorexia nervosa experience extremely low body weight combined with body image disturbance, drive for thinness, restrictive eating behaviour and often physical hyperactivity. ${ }^{1}$ Eating disorders are the third most common chronic disease in adolescents, after obesity and asthma. ${ }^{2}$ Most eating disorders begin in adolescence, and the incidence of anorexia nervosa in adolescent girls is 100 to 200 per 100000 personyears, higher than that of type 1 diabetes; ${ }^{3}$ in Europe, the lifetime prevalence anorexia nervosa is $1 \%$ to $4 \%{ }^{4}$ Treatment consists mostly of weight rehabilitation and psychotherapy, but outcomes are not satisfactory and relapse within months after the end of inpatient treatment is a common, unsolved problem.
Only one-third to one-half of patients remain weight-restored, 5 and of all mental disorders, anorexia nervosa has the highest mortality rate. ${ }^{6}$ Anorexia nervosa is also a brain-based disease: dramatic brain-volume changes in adolescents, including grey and white matter loss of up to $10 \%$, have been shown in systematic meta-analyses. ${ }^{7}$ These findings may be driven by astrocyte $\operatorname{loss}^{8}$ and appear to be largely reversible only upon weight rehabilitation. Without achieving weight recovery, brain volumes remain reduced, with potential permanent brain alterations and neuropsychological deficits that manifest in severe and enduring anorexia nervosa. ${ }^{9}$ Little is known about the underlying pathophysiology and etiology of anorexia nervosa. Specifically, we do not know why these patients lose weight and have such difficulty maintaining a healthy weight.

Correspondence to: J. Seitz, Department of Child and Adolescent Psychiatry, Psychotherapy and Psychosomatics, University Hospital, Neuenhofer Weg 21, 52074 Aachen, Germany; jseitz@ukaachen.de

Submitted Mar. 26, 2021; Revised Jun. 14, 2021; Accepted Jul. 1, 2021

Cite as: J Psychiatry Neurosci 2021 October 15;46(5). doi: 10.1503/jpn.210049 
Brain-derived neurotrophic factor (BDNF) is an important growth factor of the neurotrophin family; ${ }^{10}$ it has a strong influence on cell proliferation and differentiation, as well as on synaptic plasticity and neuronal survival. In the central nervous system, BDNF is differentially released from neurons in different brain regions (e.g., in the hippocampus and ventral tegmental area) that have been implicated in learning processes and reward ${ }^{11,12}$ - processes thought to be affected in anorexia nervosa. Furthermore, BDNF neurons in the anterior paraventricular nucleus of the hypothalamus actively inhibit appetite and food intake and enhance physical activity; those in the medial and posterior regions modulate thermogenesis. ${ }^{13}$ Consequently, there is strong evidence that BDNF interacts with eating behaviour and the regulation of body weight. Cordeira and Rios ${ }^{14}$ eliminated BDNF postnatally from mouse brains, resulting in hyperactivity and high anxiety levels, symptoms also found in acute anorexia nervosa. However, the authors also reported increased body weight and linear growth (for a recent overview, see Kumar and Singh ${ }^{15}$ ). As well, mice with only 1 BDNF allele showed increased food consumption and obesity. ${ }^{16}$ Central infusion of BDNF in rat brains led to appetite suppression and weight loss in several experiments. ${ }^{14-16}$ Thus, increased BDNF could be a risk factor for developing anorexia nervosa or inducing anorexia nervosa relapse by reducing appetite and facilitating weight loss.

Brandys and colleagues ${ }^{17}$ published a meta-analysis on BDNF and anorexia nervosa that included 7 studies and 155 patients. They found lower serum BDNF levels in a population of mostly adult patients with acute anorexia nervosa compared to healthy controls. This finding was similar to observations in other psychiatric disorders, including depression, schizophrenia and bipolar disorder. ${ }^{18-20}$ Brandys and colleagues ${ }^{17}$ interpreted this reduction as a marker of disease and possibly of ensuing brain alterations. Interestingly, body mass index (BMI) and measures of eating disorder psychopathology were positively correlated with BDNF in patients with anorexia nervosa. ${ }^{17}$

However, recent studies have painted a more ambiguous picture. Dmitrzak-Weglarz and colleagues $(n=60){ }^{21}$ Steinhäuser and colleagues $(n=77)^{22}$ and Zwipp and colleagues $(n=72)^{23}$ found no significant differences between patients with acute anorexia nervosa and healthy controls in a population consisting mostly of adolescents. Eddy and colleagues $(n=72)^{24}$ found no significant differences between adults with anorexia nervosa and healthy controls. However, reduced BDNF levels have also been confirmed in 3 studies of adolescents and adults with anorexia nervosa. ${ }^{25-27}$ Thus, it remains an open question whether BDNF is reduced in acutely ill patients with anorexia nervosa and whether findings in adolescents might differ from those in adults.

Regarding BDNF after weight recovery, 3 of 4 studies that have done cross-sectional comparisons of patients with anorexia nervosa found that patients who had recovered showed significantly higher BDNF levels than those who were acutely ill ${ }^{23,28,29}$ (but see Steinhäuser and colleagues, ${ }^{22}$ who found no difference). Five longitudinal studies of shortterm weight-recovered patients with anorexia ner- vosa $^{22,23,27-29}$ supported these findings, but only with partly trend-level findings and often small sample sizes.

Whether serum BDNF levels normalize in recovered patients with anorexia nervosa (as would be expected) or instead rise to supranormal levels that are significantly higher than that of healthy controls appears less clear. Four studies found nominal increases of $4 \%$ to $26 \%$ in recovered patients with anorexia nervosa compared to healthy controls, but these increases reached only trend level or were not significant, $^{22,23,28,29}$ while Tyszkiewicz-Nwafor and colleagues ${ }^{27}$ found that BDNF levels were still decreased after only partial weight recovery. Thus, there have been indications of an increase in serum BDNF to supranormal levels in recovered patients with anorexia nervosa, but previous studies have lacked the power to provide a definitive answer.

Supranormal BDNF levels in recovered patients with anorexia could point to elevated pre-existing BDNF levels (trait), or they could be a consequence of the illness, the weight gain or the therapeutic process (scar). Because of the anorexigenic effect of BDNF on hunger and appetite, understanding the origins of supranormal BDNF would be of great value for better understanding the pathophysiology of anorexia nervosa and could contribute to relapse prevention. ${ }^{23,29,30}$

The aim of the present study was to longitudinally and continuously measure serum BDNF in a large, well-characterized sample of adolescent girls with first-onset anorexia nervosa over a 2.5-year follow-up after inpatient treatment and to compare findings with those in healthy controls. Our hypotheses were as follows: BDNF at admission would be reduced in patients with anorexia nervosa compared to healthy controls, it would increase during treatment, and it would rise to supranormal levels compared to healthy controls during follow-up. We also explored associations between BDNF and clinical variables to identify potential interactions.

\section{Methods}

\section{Study population}

The study sample consisted of 149 female adolescent patients (aged 11 to 18 years) with first-onset anorexia nervosa and 79 age-matched healthy controls. Patients were recruited between 2007 and 2010 within the context of a multicentre study (Anorexia Nervosa Day Patient versus Inpatient Study [ANDI]) at 6 German sites, ${ }^{31}$ and, of the total 178 ANDI patients, all 149 with available blood serum samples were included. The ANDI patients with anorexia nervosa were hospitalized with a diagnosis of anorexia nervosa according to the Diagnostic and Statistical Manual of Mental Disorders IV ${ }^{32}$ and had a BMI below the 10th percentile for age.

Patients showed symptoms of anorexia nervosa for 10.13 \pm 8.02 months (mean \pm standard deviation) before admission to hospital.

Patients with organic brain disease, psychotic or bipolar disorder, substance dependence or abuse, serious selfinjurious behaviour, insufficient knowledge of the German language or an IQ of less than 85 were excluded. 
The study had 4 assessment time points. Again, we included only patients for whom serum samples had been obtained: at admission to the hospital (acutely ill patients at admission, $n=149$ ); at discharge from inpatient or 3-week inpatient plus day-patient treatment (both after short-term weight recovery), an average of 4 months after admission (patients at discharge, $n=130)$; at 1-year follow-up $(n=116)$; and at 2.5-year follow-up $(n=76)$.

We recruited female healthy controls via flyers in the Aachen area, age-matched them to the 1-year follow-up group and assessed them at a single time point. Exclusion criteria for the healthy controls were as follows: an IQ less than 85, any lifetime eating disorder or any current other psychological disorders.

Patients, controls and their parents provided written informed consent before participation. The study was implemented in alignment with the Declaration of Helsinki and Good Clinical Practice principles, including independent data management and monitoring. Local ethics committees provided their approval (EK 127/06).

\section{Blood serum collection}

Blood was collected in the morning between $0715 \mathrm{~h}$ and $0815 \mathrm{~h}$ after an overnight fast. In 30 of 79 healthy controls, blood collection was nonfasting and occurred around $1200 \mathrm{~h}$ for logistical reasons. After centrifugation and aliquoting, blood serum was stored at $-80^{\circ} \mathrm{C}$ until assessment. Serum BDNF was analyzed via enzyme-linked immunosorbent assay (ELISA) in 2 batches (2012 and 2018) using the Quantikine ELISA (R\&D Systems Inc.) at the Institute for Clinical Chemistry and Pathobiochemistry at Otto von Guericke University Magdeburg under the supervision of R.B. The BDNF measurements had intra-assay coefficients of variation of $2.4 \%$ to $3.2 \%$, inter-assay coefficients of variation of $4.3 \%$ to $7.2 \%$ and a lower limit of detection of $1.0 \mathrm{pg} / \mathrm{mL}$. Fasting and nonfasting BDNF values for the healthy controls did not differ (mean \pm standard deviation; fasting 13.61 $\pm 5.93 \mathrm{ng} / \mathrm{mL}$, nonfasting $14.83 \pm 7.84 \mathrm{ng} / \mathrm{mL}$; Student $t$ test, $p=$ $0.44)$, so we used all 79 samples in the analyses.

Because of the long time lapse between the 2 ELISA batch analyses, we compensated for possible batch and storage time effects; we also included age as potential confounder. To do this, we corrected BDNF values by calculating a multiple regression analysis, similar to previous analyses. ${ }^{23,28,33}$ We entered BDNF values for healthy controls and patients with anorexia nervosa at all 4 time points as dependent variables, and we entered batch, storage time and age as independent variables in a single joint analysis. We labelled the BDNFstandardized residuals from this analysis as "CBDNF" and used this measure for all analyses except the mixed-model analysis, which used the original BDNF results.

\section{BMI-SDS calculation}

Because normal BMI differs across development, we calculated BMI standard deviation scores (BMI-SDS) for patients and controls to obtain age-corrected information about weight development in our adolescent sample. ${ }^{34,35}$ We calculated BMI-SDS for 5 time points: baseline, before the onset of anorexia nervosa (patient recall data at the onset of weight loss), at admission, at discharge, at 1-year follow-up, and at 2.5-year follow-up. Between admission and 2.5-year followup, we measured BMI-SDS after an overnight fast; patients were wearing underwear during measurement.

\section{Psychological assessment}

To assess mental health and possible comorbidities, patients and healthy controls completed the following questionnaires: the Beck Depression Inventory II, ${ }^{36}$ the Eating Disorder Inventory 2,37 the Spence Children's Anxiety Scale ${ }^{38}$ and the Morgan-Russell Outcome Assessment Schedule. ${ }^{39}$ The MorganRussell Outcome Assessment Schedule contains following subscales: food intake, menstrual pattern, mental state, psychosexual state and socioeconomic state; the maximum possible score is 180, which means no symptoms of illness.

We used the Structured Interview for Anorexia and Bulimia Expert Interview (SIAB-EX) to assess eating disorder symptoms and typical comorbid symptoms. ${ }^{40}$ The SIAB-EX also includes a number of subscales: body image and slimness ideal, general psychopathology, sexuality and social integration, bulimic symptoms, measures to counteract weight and atypical binges. ${ }^{41}$ The maximum total score (sexuality and social integration subscales excluded) is 244 . We noted any use of antidepressant or antipsychotic medication, because these medications have been shown to increase BDNF. ${ }^{20}$

\section{Statistical analysis}

All analyses were performed using SPSS (version 23; IBM ${ }^{42}$ Two-sided $p$ values of less than 0.05 indicated statistical significance.

\section{Group comparisons of cBDNF}

First, to analyze whether cBDNF levels were different between patients with anorexia nervosa and healthy controls at the different assessment points, we compared cBDNF values between groups using Student $t$ tests. To exclude any potential effects of selective serotonin reuptake inhibitor (SSRI) use, antipsychotic medication use or anorexia nervosa subtype, we repeated this analysis excluding all patients taking an SSRI, all patients taking olanzapine and all patients of the binge-purge subtype, respectively.

\section{Longitudinal analyses of cBDNF}

To analyze longitudinal changes in the anorexia nervosa group only, we calculated a repeated-measures analysis of variance (ANOVA) of cBDNF at admission, discharge, 1-year follow-up and 2.5-year follow-up for patients with complete data at all 4 time points.

To validate the above analyses and use the full patient sample, we also assessed longitudinal changes in the anorexia nervosa group using a linear mixed model calculation with uncorrected BDNF values and independent correction for age, batch effect and storage time (this could not include healthy controls because we had data from only 1 time point for this group). We computed models with different repeated-measures 
covariance structures and compared them with likelihood ratio tests to find the best model in terms of fit and parsimony. For this procedure, we estimated models once with maximum likelihood and once with restricted maximum likelihood. Both strategies resulted in compound symmetry with a heterogeneous variance-covariance structure. We estimated the final model with restricted maximum likelihood. Our sample size allowed us to detect even small effects of $d>0.14$ with $80 \%$ power in the longitudinal ANOVA and $d>0.10$ in the mixed-model analyses. We repeated both analyses with restrictive-subtype patients only.

\section{Parameters influencing cBDNF}

To identify any potential influences of clinical parameters on the longitudinal course of BDNF, we repeated the linear mixed model described above. We included the following at each time point as independent variables, separately for each influencing factor: BMI-SDS, SSRI use, olanzapine use, anorexia nervosa subtype, menstruation status, hormonal contraceptive use and total scores on the Beck Depression Inventory II, Spence Children's Anxiety Scale, Morgan-Russell Outcome Assessment Schedule, Eating Disorder Inventory 2 and SIAB-EX.

To further analyze which variables could influence cBDNF at each time point, we used backward linear regression analyses, including the following potential influencing factors from the same or previous time points: BMI-SDS, weight loss before admission, illness duration, and total scores on the Beck Depression Inventory II, Morgan-Russell Outcome Assessment Schedule, Spence Children's Anxiety Scale and SIAB-EX. For a full list of included parameters, please see Appendix 1, available at jpn.ca.
Influence of cBDNF on weight development and eating disorder psychopathology

We also determined whether prior cBDNF measurement would help predict BMI-SDS or SIAB-EX total score at a later time point.

First, we performed 3 backward linear regressions using BMI-SDS as the dependent variable and previous cBDNF measurements, illness duration, current BMI-SDS, previous weight change and questionnaire data from previous assessment points as independent variables.

Then, we calculated whether previous cBDNF measurements would predict SIAB-EX total score at a later time point using the same strategy, using SIAB-EX as the dependent variable and CBDNF, previous BMI-SDS variables and illness duration as independent variables. Because of high intercorrelation, we did not include data from the other questionnaires.

Finally, we tested whether cBDNF could help predict relapse, conceptualized as underweight (BMI less than the 10th percentile or BMI-SDS less than -1.28) at 1-year and 2.5-year follow-up using logistical regression analysis with all previous cBDNF measurements as independent variables. We further compared previous cBDNF values of relapsed patients with those of non-relapsed patients at 1-year and 2.5-year follow-up using Student $t$ tests.

\section{Results}

For participant clinical characteristics and BDNF and cBDNF values, see Table 1 . The results are reported as mean \pm standard deviation.

\begin{tabular}{|c|c|c|c|c|c|}
\hline \multirow[b]{2}{*}{ Characteristic } & \multirow[b]{2}{*}{$\begin{array}{c}\text { Healthy } \\
\text { controls } \\
n=79\end{array}$} & \multicolumn{4}{|c|}{ Patients with anorexia nervosa } \\
\hline & & $\begin{array}{l}\text { At admission } \\
n=149\end{array}$ & $\begin{array}{l}\text { At discharge } \\
\quad n=130\end{array}$ & $\begin{array}{c}\text { At 1-year } \\
\text { follow-up } \\
n=116\end{array}$ & $\begin{array}{c}\text { At 2.5-year } \\
\text { follow-up } \\
n=76\end{array}$ \\
\hline Age, yr & $16.35 \pm 1.93$ & $15.32 \pm 1.46$ & $15.56 \pm 1.51$ & $16.3 \pm 1.49$ & $17.57 \pm 1.54$ \\
\hline $\mathrm{BMI}, \mathrm{kg} / \mathrm{m}^{2}$ & $21.25 \pm 2.75$ & $15.03 \pm 1.28$ & $18.08 \pm 0.99$ & $18.21 \pm 1.69$ & $19.26 \pm 2.4$ \\
\hline BMI-SDS & $0.02 \pm 0.81$ & $-2.68 \pm 0.91$ & $-0.96 \pm 0.42$ & $-1.11 \pm 0.76$ & $-0.83 \pm 1.14$ \\
\hline >10th age-adapted BMI percentile* & $100 \%$ & $3.35 \%$ & $83.7 \%$ & $68.42 \%$ & $71.05 \%$ \\
\hline Eating Disorder Inventory 2 score & $186.22 \pm 44.19$ & $261.41 \pm 59.21$ & $235.83 \pm 61.27$ & $256.05 \pm 72.09$ & $251.66 \pm 63.14$ \\
\hline Beck Depression Inventory II score & $5.07 \pm 5.26$ & $19.54 \pm 10.38$ & $10.46 \pm 8.44$ & $13.36 \pm 11.06$ & $12.63 \pm 10.81$ \\
\hline Spence Children's Anxiety Scale score & $15.11 \pm 7.7$ & $22.53 \pm 12.81$ & $16.92 \pm 11.85$ & $18.28 \pm 11.98$ & $20.2 \pm 14.91$ \\
\hline Morgan-Russell Outcome Assessment Schedule score & - & $84.31 \pm 25.11$ & $97.47 \pm 32.93$ & $116.77 \pm 28.86$ & $125.09 \pm 23.61$ \\
\hline SIAB-EX total score & - & $63.74 \pm 17.64$ & $34.56 \pm 14.42$ & $40.57 \pm 22.1$ & $37.04 \pm 24.19$ \\
\hline Binge-purge subtype & - & $19.5 \%$ & $19.2 \%$ & $17.2 \%$ & $23.7 \%$ \\
\hline Taking SSRIs & - & $5 \%$ & $10 \%$ & $16 \%$ & $18 \%$ \\
\hline Taking olanzapine & - & $0 \%$ & $7.4 \%$ & $6 \%$ & $0 \%$ \\
\hline Menstruating $†$ & - & $0 \%$ & $18.3 \%$ & $31.1 \%$ & $38.7 \%$ \\
\hline Taking hormonal contraception & - & $11.5 \%$ & $8.3 \%$ & $13.1 \%$ & $48.0 \%$ \\
\hline Serum BDNF, ng/mL & $14.08 \pm 0.75$ & $17.36 \pm 6.57$ & $18.42 \pm 7.48$ & $19.07 \pm 7.59$ & $20.84 \pm 8.26$ \\
\hline cBDNF & $-0.44 \pm 6.92$ & $-1.76 \pm 6.24$ & $-0.31 \pm 6.96$ & $1.07 \pm 7.11$ & $2.8 \pm 8.04$ \\
\hline \multicolumn{6}{|c|}{$\begin{array}{l}\text { BDNF = brain-derived neurotrophic factor; } \mathrm{BMI}=\text { body mass index; BMI-SDS = BMI standard deviation score (age- } \\
\text { batch effect; } \mathrm{SIAB}-\mathrm{EX}=\text { Structured Interview for Anorexia and Bulimia expert interview; SSRI = selective serotonin } \\
\text { Values are mean } \pm \text { standard deviation or percent. } \\
{ }^{*} \text { Percentage of patients who had recovered a normal BMI. } \\
\dagger \text { Percentage of patients with regular or irregular menstruation, excluding patients taking hormonal contraception. }\end{array}$} \\
\hline
\end{tabular}




\section{Group comparisons of cBDNF}

We found that the cBDNF levels of patients with anorexia nervosa at admission were only nominally lower than those of healthy controls $(p=0.15)$. cBDNF levels did not differ at discharge $(p=0.90)$ or at 1 -year follow-up $(p=0.14)$, but they were increased significantly at 2.5-year follow-up ( $p=0.008$; see Figure 1 and Appendix 1, Table S1).

\section{Longitudinal changes in $C B D N F$}

cBDNF levels in patients with anorexia nervosa increased continuously from hospital admission until 2.5-year followup, as evidenced by repeated-measures ANOVA (all post hoc tests $p<0.05$ except for patients at admission versus patients at discharge; see Figure 1 and Appendix 1, Table S2).

Our mixed-model analysis in only patients with anorexia nervosa showed that when we calculated the model using uncorrected BDNF and correcting for age, batch and storage time separately, we found significant time effects of rising BDNF between all 4 time points, except between discharge and 1-year follow-up (see Appendix 1, Figure S1, and Table S3). The exclusion of patients taking an SSRI or olanzapine, or of patients of the binge-purge subtype, did not significantly alter group comparisons or longitudinal results (for those with restrictive anorexia nervosa only, see Appendix 1, Tables S4 to S6).

\section{Parameters influencing $c B D N F$}

We found no significant longitudinal influences on cBDNF when we separately added the following as independent variables to the mixed-model analysis above: BMI-SDS, SSRI use, olanzapine use, menstruation status, hormonal contraceptives or total scores on the Beck Depression Inventory II, Spence Children's Anxiety Scale, Morgan-Russell Outcome Assessment Schedule, Eating Disorder Inventory 2 or SIAB-EX.

We also conducted linear regression analyses for factors associated with cBDNF at each time point separately. We found that cBDNF at discharge was significantly inversely associated with eating psychopathology (SIAB-EX at discharge;

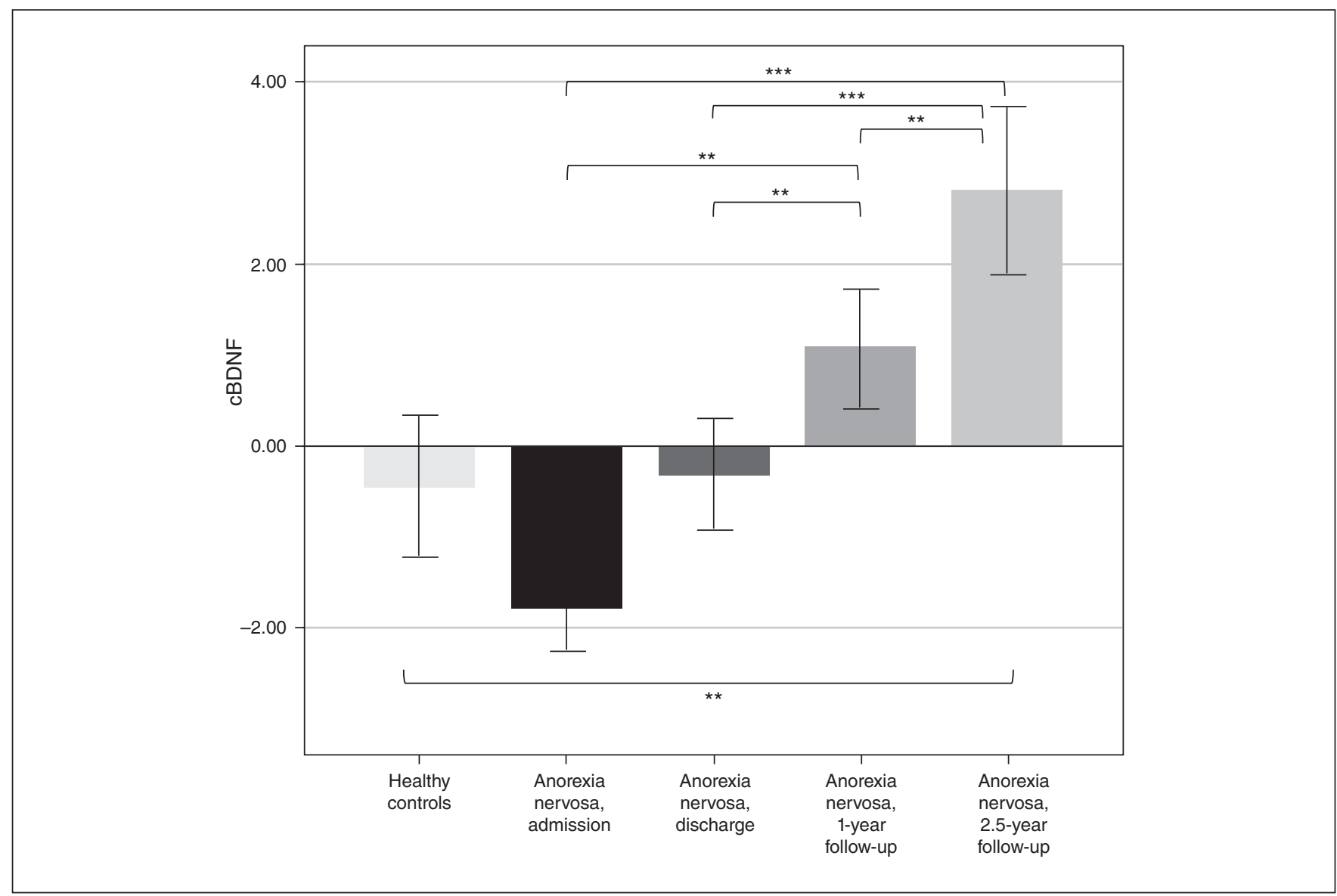

Figure 1: cBDNF levels in healthy controls $(n=79)$ and in patients with anorexia nervosa at admission $(n=149)$, at discharge $(n=130)$, at 1-year follow-up $(n=116)$, and at 2.5-year follow-up $(n=76)$. Lower bracket: Student $t$ test of healthy controls versus patients with anorexia at different time points; only healthy controls versus patients with anorexia nervosa at 2.5-year follow-up reached significance. Upper brackets: post hoc tests, repeated-measures analysis of variance in 68 patients with anorexia nervosa using available data at all time points. ${ }^{* *} p<0.01$; ${ }^{* * *} p<0.001$. cBDNF = brain-derived neurotrophic factor corrected for age, storage time and batch effect. 
$\left.F=7.201, R^{2}=0.071, \beta=-0.267, p=0.009\right)$, and cBDNF at 1-year follow-up was associated with previous weight gain between discharge and 1-year follow-up (change in BMI-SDS [discharge - 1-year follow-up]; $F=4.058, R^{2}=0.038, \beta=$ 0.195, $p=0.047$; Figure 2).

Our tests of whether cBDNF could help predict BMI-SDS or eating psychopathology (SIAB-EX total score) based on backward linear regressions revealed no significant effects of cBDNF, nor did cBDNF significantly predict relapse in the logistical regression analysis. Interestingly, previous cBDNF values were nominally higher in relapsed patients, but the difference compared to nonrelapsed patients did not reach significance (cBDNF at discharge: $-0.33 \pm 7.09$ [relapse at 1 year, $n=40$ ] versus $-1.35 \pm 6.87$ [no relapse at 1 year, $n=74$ ], $p>0.05$; cBDNF at 1 year: $2.07 \pm 6.13$ [relapse at 2.5 years, $n=21$ ] versus $0.58 \pm 7.69$ [no relapse at 2.5 years, $n=53], p>0.05)$.

\section{Exploratory analysis}

We conducted an exploratory Pearson correlation analysis of the remaining time points to check for further associations between CBDNF and either BMI-SDS or SIAB-EX total score, which were significant in the regression analyses above. This analysis revealed additional associations between previous SIAB-EX and cBDNF levels: change in SIAB-EX (discharge admission) was negatively correlated with cBDNF at discharge $(r=-0.281, p=0.002)$ and SIAB-EX at 1-year followup was negatively correlated with cBDNF at 2.5-year follow-up ( $r=-0.354, p=0.002$, see Appendix 1, Figure S2).

\section{Discussion}

The aim of this study was to observe serum BDNF development in the largest longitudinal sample to date (consisting of 149 female adolescent patients with first-time onset anorexia nervosa) over a period of 2.5 years, and to compare these findings to those for 79 age-matched healthy controls. At admission, cBDNF levels in patients showed only nominally lower values compared to those of healthy controls, and then cBDNF values increased continuously to supranormal levels at 2.5-year follow-up. cBDNF values were inversely associated with eating disorder psychopathology (SIAB-EX total score) at discharge and positively associated with previous weight gain at 1-year follow-up.

Increased cBDNF levels at 2.5 years after first hospitalization is a new and important finding. Because BDNF has a known anorexigenic effect on appetite and satiety - in addition to potentially positive effects on neuronal growth and synapse formation - it might make patients more susceptible to weight loss and relapse.

The nominally lower cBDNF values we found at admission point in the same direction as a previous, similarly powered meta-analysis by Brandys and colleagues, ${ }^{17}$ which found significantly lower serum BDNF values in 155 mostly adult patients with acute anorexia nervosa compared to healthy controls. However, 4 recent studies did not find significant differences in a total of 209 adolescents and 72 adults $^{21-24}$ (but see divergent results ${ }^{25-27}$ ). This raises the possibility that BDNF reduction might not be as pronounced in adolescent patients with anorexia nervosa, who tend to be less chronically ill. We could neither support nor dismiss this explanation, because we found no correlation between cBDNF and duration of illness in our study. However, this finding might have been because of a lack of variability of illness duration in this cohort of first-onset patients and definitely warrants further research. As mentioned above, lower BDNF levels have also been reported in other chronic mental disorders, including schizophrenia, bipolar disorder and depression. ${ }^{18-20}$ BDNF plays an important role as a growth factor in terms of long-term potentiation and synaptic plasticity, which have been shown to be altered in these diseases. ${ }^{43,44}$ It could certainly also be true for anorexia nervosa, in which striking brain volume reduction, astrocyte reduction, neuron-associated light chain protein deviations and (moderate) neuropsychological deficits have been well documented.8,9,45,46

Our results showed that BDNF levels increased continuously to supranormal values over 2.5 years of observation in patients with anorexia nervosa. The increase in BDNF over the course of weight rehabilitation and recovery fits well with the pre-existing literature. ${ }^{22,23,27-29}$ This increase has been interpreted by previous authors as a reconstruction process, indicating repair mechanisms and reversal of damage induced by starvation. ${ }^{17,23}$ However, this explanation does not appear to capture the whole process, because it does not explain our new finding of a supranormal endpoint at 2.5-year follow-up. Indeed, BMI-SDS and eating disorder psychopathology did not change much, on average, between discharge and 2.5-year follow-up in our study, disputing a continuous process of repair and improvement or a rebound effect. On the other hand, the possibility of truly increased levels of BDNF after recovery has been hinted at by previous studies, ${ }^{22,23,28,29}$ which found that BDNF values were increased by $4 \%$ to $25 \%$ above those of controls. However, these results did not reach significance, partially because of limited power $(n=18-62)$. In the present study, we found significantly increased cBDNF, even in a mixed sample of patients who had recovered and were still battling with disease, making our result relatively stronger.

We were unable to show significant associations between cBDNF levels and BMI-SDS in general or with underweight status at 1-year and 2.5-year follow-up, making a simple relationship between cBDNF and starvation or body weight recovery unlikely. However, predating cBDNF levels at discharge and 1 year were both nominally higher in later relapsed patients compared to nonrelapsed patients, compatible with the role of $\mathrm{CBDNF}$ as a risk factor for relapse. Two overlapping effects could have been at play: on one hand, cBDNF could be a (positive) marker of brain repair processes during acute weight restoration; on the other hand, increased cBDNF levels after weight restoration could be a (negative) marker for a higher risk of relapse.

Several confounders could influence serum BDNF over time. For example, the increase could be an effect of time, because BDNF is known to increase with age. ${ }^{47,48}$ However, we corrected cBDNF for age and included age in the mixed-model 
A

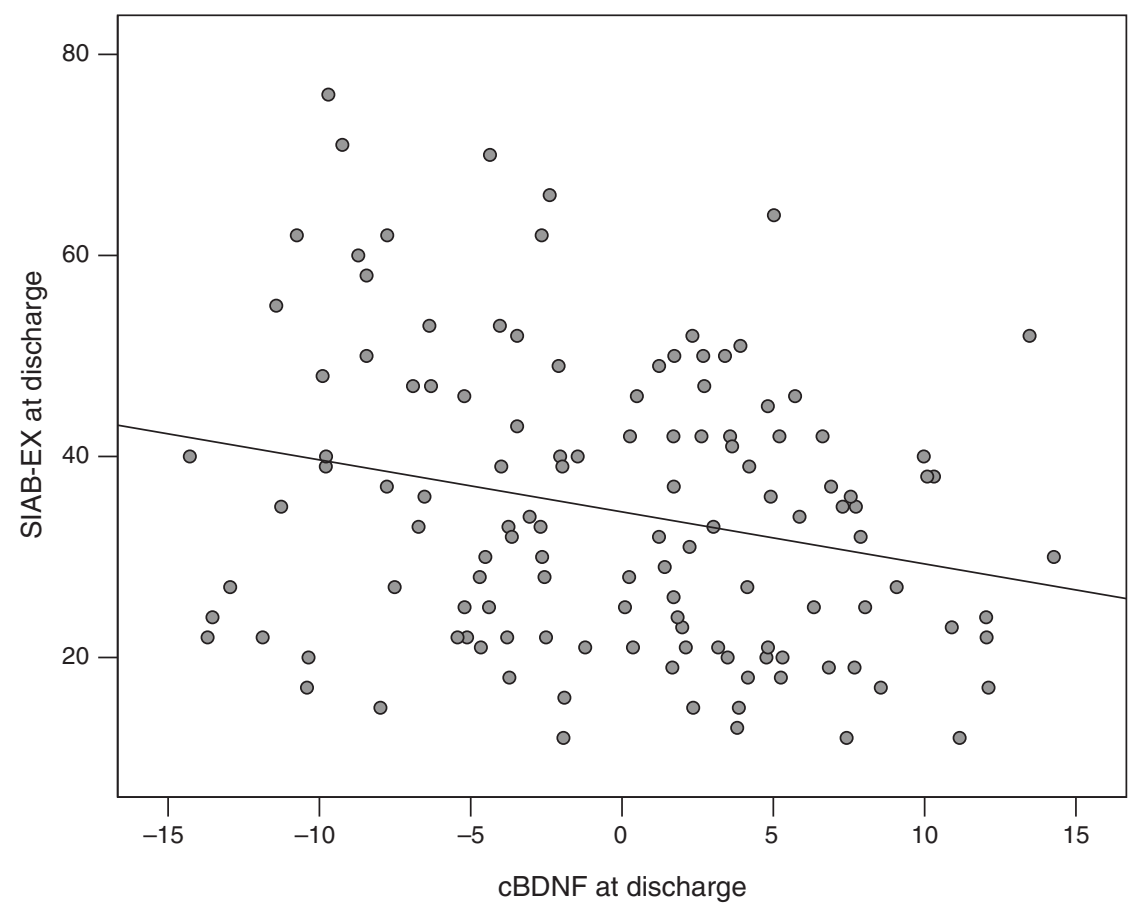

B

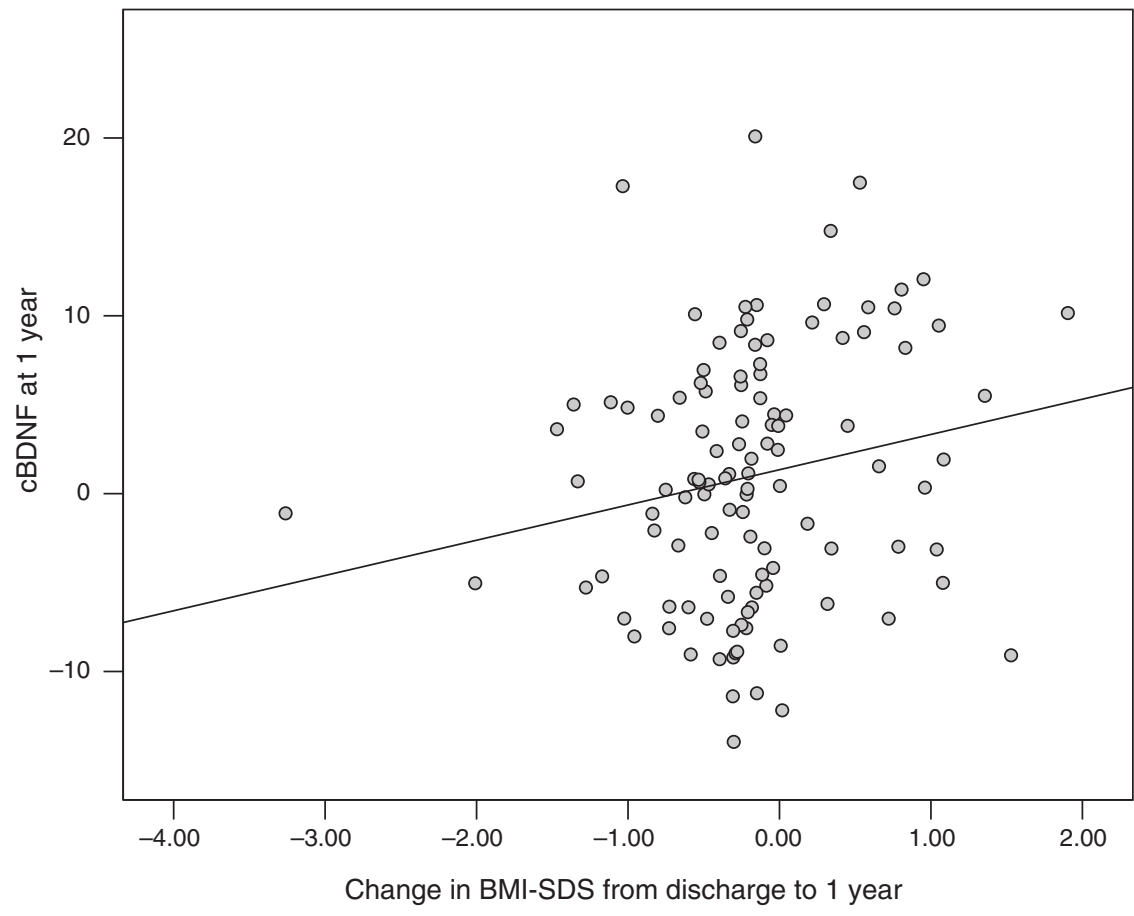

Figure 2: Bivariate scatterplots of factors that significantly influenced cBDNF in the regression analysis. (A) Negative association between cBDNF and SIAB-EX at discharge. (B) Increased BMI-SDS from discharge to 1-year follow-up predicted cBDNF at 1-year follow-up. Spearman $\rho$ correlation, 2-tailed. ${ }^{*} p<0.05$. BMI-SDS = body mass index standard deviation score (age-adapted BMI); $\mathrm{cBDNF}=$ brain-derived neurotrophic factor corrected for age, storage time and batch effect; SIAB-EX = Structured Interview for Anorexia and Bulimia Expert Interview. 
analysis, where it was not significant, so an effect of time was not likely. Exercise also has a positive effect on BDNF excretion in patients, healthy controls and animals. ${ }^{49-51}$ Theoretically, an increase in exercise over time in our previous patients could have influenced BDNF values. Because we did not include objective exercise measures, we could not rule out this effect.

We are aware that BDNF does not pass the blood-brain barrier because of its size, ${ }^{52,53}$ so peripheral BDNF levels do not directly influence synaptic plasticity during recovery. However, multiple studies have suggested a strong association between BDNF levels in the blood and the brain (for example, see Klein and colleagues ${ }^{54}$ and Cao and colleagues ${ }^{55}$ ); our hypotheses about BDNF in the brain and abnormal eating behaviour are based on these findings.

A possible underlying mechanism for rising BDNF levels could be abnormalities in estrogen-signalling pathways in anorexia. Sohrabji and colleagues ${ }^{56}$ reported that estrogen replacement in rats led to increasing BDNF expression in several brain regions. Wessels and colleagues ${ }^{57}$ showed that treatment with estradiol in ovariectomized mice significantly increased BDNF in the mouse uterus, and Cui and colleagues ${ }^{58}$ showed behavioural disturbances in mice deficient in estrogen-related receptor $\alpha$. Increasing blood levels of estrogen, provoking an aberrant brain response during puberty, could be involved in anorexia nervosa and explain why mainly young women are affected by anorexia nervosa. ${ }^{59}$

Another possible underlying mechanism for elevated BDNF levels is linked to leptin, which is strongly reduced in patients with anorexia nervosa. ${ }^{60} \mathrm{Li}$ and colleagues ${ }^{61}$ suggested that BDNF-expressing neurons were targets of leptin, and that leptin deficiency decreased BDNF gene expression. Milos and colleagues ${ }^{62}$ treated 3 female patients off-label with metreleptin and observed decreasing hyperactivity and decreasing weight phobia in 2 patients and improved mood in all 3 patients. Finally, antidepressant medication has been shown to increase reduced BDNF values in depressive patients, sometimes up to normalization..$^{20}$ Because of the high comorbidity of depression and anorexia nervosa, antidepressant use could have played an important role in our cohort, but it was not a significant covariate in the mixed model. We excluded all patients taking antidepressant or antipsychotic medications, repeated the analyses and had similar results, making it unlikely that antidepressants were confounders. ${ }^{23}$

Interestingly, patients with autism spectrum disorder also show increased levels of BDNF, as shown in 2 large metaanalyses with more than 1200 and 1700 participants, respectively. ${ }^{63,64}$ Because patients with anorexia nervosa are known to portray more autistic traits, ${ }^{65}$ a common developmental pathophysiologic mechanism in the brain could be at work. Many patients with autism also tend to be "picky eaters," 66 and high-functioning (but not low-functioning) patients with autism are also known to weigh less and have a higher chance of being underweight, ${ }^{67-69}$ pointing to a possible eating-related effect of increased BDNF in autism and warranting future investigation.
Because BDNF has pleiotropic functions that differ in varying tissues and states in patients with anorexia nervosa, any overlapping effects of the above-described (positive) initial repair mechanisms, altered effects on brain development and potentially negative endpoint effects on hunger suppression do not exclude each other. Truly increased levels of BDNF could predispose patients to future weight loss because of its hunger-suppressing properties, or even represent a predisposing factor (trait) for anorexia nervosa if increased BDNF is present before the illness. ${ }^{23,29}$

In our association analysis, we found that lower eatingdisorder psychopathology and higher weight gain were associated with higher cBDNF. This fit well with the literature, in which several studies have found BDNF correlations with $\mathrm{BMI}^{28,70-72}$ and illness markers such as Eating Attitude Test subscales, ${ }^{72}$ Eating Disorder Inventory subscales and the Global Severity Index. ${ }^{28}$ Nevertheless, we were surprised not to find more associations, given the power of this large longitudinal study. The pleiotropic, partly antagonistic functions of BDNF and the multiple factors influencing them ${ }^{47}$ could add too much unexplained variation to detect further determining factors.

For this reason, the solid finding of supranormal cBDNF at 2.5-year follow-up must be valued even more highly. Future research is needed to analyze the causal underpinnings (for example, by longer-term animal experiments with follow-up after starvation) and discern the repair and scarring hypotheses. As well, serum BDNF levels in high-risk groups (such as healthy siblings of patients with anorexia nervosa or preadolescent offspring of former patients with anorexia nervosa) could be compared with serum BDNF levels in healthy controls to identify whether increased BDNF might be a genetic trait. If corroborated, increased BDNF in groups at high risk for anorexia nervosa might have the potential to become a clinical marker of predisposition. Interestingly, Mercader and colleagues ${ }^{73}$ analyzed plasma BDNF and found significantly increased levels in 21 patients with anorexia nervosa compared to their siblings. Unfortunately, they did not analyze BDNF in unrelated healthy controls.

\section{Limitations}

A limiting factor of the present study was substantial attrition: the number of participants who provided blood serum samples decreased over time. However, similar losses to followup are common in longitudinal research. ${ }^{74}$ To ensure that we could still use data from all patients longitudinally, we conducted an additional mixed-model analysis to include all available information at each point of measurement. We also compared patients who were lost to follow-up with those who had complete data. Patients who were lost to follow-up were slightly older and had a longer illness duration, a lower BMI-SDS and Morgan-Russell Outcome Assessment Schedule score, but slightly higher BDNF and cBDNF values at admission (Appendix 1, Table S7). However, losing patients with higher initial BDNF values would be expected to lower the group mean, strengthening our finding of a continuous increase and elevated cBDNF levels at 2.5-year follow-up in the 
remaining participants. For logistical reasons, we had to analyze blood serum samples in 2 different ELISA runs at different time points, and this introduced a risk of batch and storage time effects. Indeed, we found a significant batch effect, but no effect of additional storage time; however, we corrected all calculations for this confounding factor. Finally, we evaluated healthy controls at only 1 time point, so they could not be included in the mixed model. However, we were able to compare each time point separately after correcting for age.

\section{Conclusion}

To our knowledge, this is the largest and longest longitudinal study of BDNF development in adolescent first-time patients with anorexia nervosa to date. It answers the important question of whether BDNF normalizes or increases above values in healthy controls over time. We showed that cBDNF levels rose continuously in patients with anorexia nervosa over treatment and long-term follow-up, leading to significantly elevated cBDNF compared to healthy controls after 2.5 years. Furthermore, we found inverse associations between cBDNF and eating disorder psychopathology and positive correlations between $\mathrm{cBDNF}$ and previous weight gain. Supranormal BDNF 2.5 years after first-onset anorexia nervosa may have come from a reinstated pre-existing elevation (state) or the long-term consequences of the disease or rehabilitation period (scar). The anorectic effect of BDNF on hunger and food consumption could contribute to pathophysiological models of the initial development of eating disorders and to the prevention of relapse. Further research should explore the underlying mechanisms and causality of our observations.

Acknowledgments: We especially want to thank all patients who participated in the ANDI study, as well as their parents. We also want to acknowledge all staff who took part in caring for these patients and supporting the ANDI study. Funding for the ANDI study and these serum analyses were provided by the German Ministry for Education and Research (grants 01GV0602 and 01GV0623).

Affiliations: From the Department of Child and Adolescent Psychiatry, Psychotherapy and Psychosomatics, University Hospital, RWTH University Aachen, Germany (Borsdorf, Dahmen, Buehren, Scharke, Herpertz-Dahlmann, Seitz); the kbo-Heckscher Klinikum, Academic Teaching Hospital, Ludwig Maximilian University, Munich, Germany (Buehren); the Institute of Medical Informatics and Statistics, Kiel University, Germany (Dempfle); the Department of Child and Adolescent Psychiatry, Psychosomatics and Psychotherapy, University Hospital, Wuerzburg, Germany (Egberts); the Translational Developmental Neuroscience Section, Division of Psychological and Social Medicine and Developmental Neurosciences, Faculty of Medicine, TU Dresden, Germany (Ehrlich); the Eating Disorders Research and Treatment Center, Department of Child and Adolescent Psychiatry, Faculty of Medicine, TU Dresden, Germany (Ehrlich); the Department of Child and Adolescent Psychiatry and Psychotherapy, University Medical Center Freiburg, Germany (Fleischhaker); the Child Neuropsychology Section, Department of Child and Adolescent Psychiatry, Psychotherapy and Psychosomatics, University Hospital, RWTH University Aachen (Konrad); the JARA-Institute Molecular Neuroscience and Neuroimaging (INM-11), Juelich Research Centre, Germany (Konrad); the Oberberg Fachklinik Konraderhof, Cologne-Huerth, Germany (Schwarte); the Institute of Medical Biometry and Epidemiology, Philipps-University Marburg, Germany (Timmesfeld); the Department of Medical Informatics, Bio- metrics and Epidemiology, Ruhr University Bochum, Germany (Timmesfeld); the Department of Child and Adolescent Psychiatry and Psychotherapy, Cologne City Hospitals, Germany (Wewetzer); the Institute of Laboratory Medicine, Clinical Chemistry and Molecular Diagnostics, University of Leipzig, Germany (Biemann); the Cognitive and Experimental Psychology, Institute of Psychology, RWTH Aachen University, Germany (Scharke).

Competing interests: K. Egberts reports membership in the ANDI-Konsortium. N. Timmesfeld reports grants from Bundesministerium für Bildung und Forschung during the conduct of the study. No other competing interests declared.

Contributors: B. Borsdorf, K. Konrad, N. Timmesfeld, B. HerpertzDahlmann and J. Seitz designed the study. B. Dahmen, K. Buehren, K. Egberts, S. Ehrlich, C. Fleischhaker, R. Schwarte and C. Wewetzer acquired the data, which B. Borsdorf, A. Dempfle, S. Ehrlich, R. Biemann, W. Scharke, B. Herpertz-Dahlmann and J. Seitz analyzed. B. Borsdorf wrote the article, which all authors reviewed. All authors approved the final version to be published and can certify that no other individuals not listed as authors have made substantial contributions to the paper.

Content licence: This is an Open Access article distributed in accordance with the terms of the Creative Commons Attribution (CC BY-NC-ND 4.0) licence, which permits use, distribution and reproduction in any medium, provided that the original publication is properly cited, the use is noncommercial (i.e., research or educational use), and no modifications or adaptations are made. See: https:// creativecommons.org/licenses/by-nc-nd/4.0/

\section{References}

1. American Psychiatric Association. Diagnostic and statistical manual of mental disorders. 5th ed. Arlington (VA): American Psychiatric Association; 2013.

2. Herpertz-Dahlmann B. Adolescent eating disorders: update on definitions, symptomatology, epidemiology, and comorbidity. Child Adolesc Psychiatr Clin N Am 2015;24:177-96.

3. Javaras KN, Runfola CD, Thornton LM, et al. Sex- and age-specific incidence of healthcare-register-recorded eating disorders in the complete Swedish 1979-2001 birth cohort. Int J Eat Disord 2015;48: 1070-81.

4. Keski-Rahkonen A, Mustelin L. Epidemiology of eating disorders in Europe: prevalence, incidence, comorbidity, course, consequences, and risk factors. Curr Opin Psychiatry 2016;29:340-5.

5. Herpertz-Dahlmann B. Treatment of eating disorders in child and adolescent psychiatry. Curr Opin Psychiatry 2017;30:438-445.

6. Hoang U, Goldacre M, James A. Mortality following hospital discharge with a diagnosis of eating disorder: national record linkage study, England, 2001-2009. Int J Eat Disord 2014;47:507-15.

7. Seitz J, Konrad K, Herpertz-Dahlmann B. Extend, pathomechanism and clinical consequences of brain volume changes in anorexia nervosa. Curr Neuropharmacol 2018;16:1164-73.

8. Frintrop L, Liesbrock J, Paulukat L, et al. Reduced astrocyte density underlying brain volume reduction in activity-based anorexia rats. World J Biol Psychiatry 2018;19:225-35.

9. Wentz E, Dobrescu SR, Dinkler L, et al. Thirty years after anorexia nervosa onset, serum neurofilament light chain protein concentration indicates neuronal injury. Eur Child Adolesc Psychiatry 2020;s00787-020-01657-7.

10. Nagahara AH, Tuszynski MH. Potential therapeutic uses of BDNF in neurological and psychiatric disorders. Nat Rev Drug Discov 2011;10: 209-19.

11. Homan P, Grob S, Milos G, et al. The role of BDNF, leptin, and catecholamines in reward learning in bulimia nervosa. Int $J$ Neuropsychopharmacol 2014;18:pyu092.

12. Kowiańskin P, Lietzau G, Czuba E. BDNF: A key factor with multipotent impact on brain signaling and synaptic plasticity. Cell Mol Neurobiol 2018;38:579-93. 
13. An JJ, Liao GY, Kinney CE, et al. Discrete BDNF neurons in the paraventricular hypothalamus control feeding and energy expenditure. Cell Metab 2015;22:175-88.

14. Cordeira J, Rios M. Weighing in the role of BDNF in the central control of eating behavior. Mol Neurobiol 2011;44:441-8.

15. Kumar U, Singh S. Role of somatostatin in the regulation of central and peripheral factors of satiety and obesity. Int J Mol Sci 2020;21: 2568.

16. Xu B, Xie X. Neurotrophic factor control of satiety and body weight. Nat Rev Neurosci 2016;17:282-92.

17. Brandys MK, Kas MJ, van Elburg AA, et al. A meta-analysis of circulating BDNF concentrations in anorexia nervosa. World J Biol Psychiatry 2011;12:444-54.

18. Brand SJ, Moller M, Harvey BH. A review of biomarkers in mood and psychotic disorders: a dissection of clinical vs. preclinical correlates. Curr Neuropharmacol 2015;13:324-68.

19. Fernandes BS, Steiner J, Berk M, et al. Peripheral brain-derived neurotrophic factor in schizophrenia and the role of antipsychotics: meta-analysis and implications. Mol Psychiatry 2015;20:1108-19.

20. Molendijk ML, Spinhoven P, Polak M, et al. Serum BDNF concentrations as peripheral manifestations of depression: evidence from a systematic review and meta-analyses on 179 associations (N=9484). Mol Psychiatry 2014;19:791-800.

21. Dmitrzak-Weglarz M, Skibinska M, Slopien A, et al. Serum neurotrophin concentrations in Polish adolescent girls with anorexia nervosa. Neuropsychobiology 2013;67:25-32.

22. Steinhäuser JL, King JA, Tam FI, et al. Is serum BDNF altered in acute, short- and long-term recovered restrictive type anorexia nervosa? Nutrients 2021;13:432.

23. Zwipp J, Hass J, Schober I, et al. Serum brain-derived neurotrophic factor and cognitive functioning in underweight, weight-recovered and partially weight-recovered females with anorexia nervosa. Prog Neuropsychopharmacol Biol Psychiatry 2014;54:163-9.

24. Eddy KT, Lawson EA, Meade C, et al. Appetite regulatory hormones in women with anorexia nervosa: binge-eating/purging versus restricting type. J Clin Psychiatry 2015;76:19-24.

25. Dalton B, Campbell IC, Chung R, et al. Inflammatory markers in anorexia nervosa: an exploratory study. Nutrients 2018;10:1573.

26. Mancuso C, Izquierdo A, Slattery $M$, et al. Changes in appetiteregulating hormones following food intake are associated with changes in reported appetite and a measure of hedonic eating in girls and young women with anorexia nervosa. Psychoneuroendocrinology 2019;113:104556

27. Tyszkiewicz-Nwafor M, Rybakowski F, Dmitrzak-Weglarz M, et al. Brain-derived neurotrophic factor and oxytocin signaling in association with clinical symptoms in adolescent inpatients with anorexia nervosa—a longitudinal study. Front Psychiatry 2019;10:1032.

28. Ehrlich S, Salbach-Andrae H, Eckart S, et al. Serum brain-derived neurotrophic factor and peripheral indicators of the serotonin system in underweight and weight-recovered adolescent girls and women with anorexia nervosa. J Psychiatry Neurosci 2009;34:323-9.

29. Nakazato $M$, Tchanturia $K$, Schmidt $U$, et al. Brain-derived neurotrophic factor (BDNF) and set-shifting in currently ill and recovered anorexia nervosa (AN) patients. Psychol Med 2009;39:1029-35.

30. Nakazato M, Hashimoto K, Shimizu E, et al. Possible involvement of brain-derived neurotrophic factor in eating disorders. IUBMB Life 2012;64:355-61.

31. Herpertz-Dahlmann B, Schwarte R, Krei M, et al. Day-patient treatment after short inpatient care versus continued inpatient treatment in adolescents with anorexia nervosa (ANDI): a multicentre, randomised, open-label, non-inferiority trial. Lancet 2014;383:1222-9.

32. American Psychiatric Association. Diagnostic and statistical manual of mental disorders. 4th ed, text revision. Arlington (VA): American Psychiatric Association; 2000.

33. Nassan M, Veldic M, Winham S, et al. Methylation of brain derived neurotrophic factor (BDNF) Val66Met CpG site is associated with early onset bipolar disorder. J Affect Disord 2020;267:96-102.
34. Ped (z) pediatric calculator. Leipzig, Germany: Daniel Gräfe; 2008-2021. Available: www.pedz.de/de/impressum.html (accessed 2020 May 2).

35. Rosario AS, Kurth B-M, Stolzenberg H, et al. Body mass index percentiles for children and adolescents in Germany based on a nationally representative sample (KiGGS 2003-2006). Eur J Clin Nutr 2010;64:341-9.

36. Hautzinger M, Keller F, Beck AT, et al. Beck depressions-inventar II: manual. San Antonio (TX): Pearson Assessment; 2009.

37. Paul T, Thiel A. Eating disorder inventory 2, Deutsche version: manual. Boston: Hogrefe; 2005.

38. Spence SH, Barrett PM, Turner CM. Psychometric properties of the Spence Children's Anxiety Scale with young adolescents. J Anxiety Disord 2003;17:605-25.

39. Morgan HG, Hayward AE. Clinical assessment of anorexia nervosa. The Morgan-Russell outcome assessment schedule. $\mathrm{Br} J$ Psychiatry 1988;152:367-71.

40. Fichter M, Quadflieg N. Das Strukturierte Interview für Anorektische und Bulimische Ess-Störungen nach DSM-IV und ICD-10 zur Expertenbeurteilung (SIAB-EX) und dazugehöriger Fragebogen zur Selbsteinschätzung (SIAB-S). In: Verhaltenstherapie. No. 4. Freiburg, Germany: S. Karger; 2001; 314-25.

41. Fichter M, Herpertz S, Quadflieg N, et al. Structured interview for anorexic and bulimic disorders for DSM-IV and ICD-10: updated (third) revision. Int J Eat Disord 1998;24:227-49.

42. SPSS statistics for Windows, version 23.0. Armonk (NY): IBM Corp.; 2015.

43. Cattaneo A, Cattane N, Begni V, et al. The human BDNF gene: peripheral gene expression and protein levels as biomarkers for psychiatric disorders. Transl Psychiatry 2016;6:e958.

44. Noble EE, Billington CJ, Kotz CM, et al. The lighter side of BDNF. Am J Physiol Regul Integr Comp Physiol 2011;300:R1053-69.

45. King JA, Frank GKW, Thompson PM, et al. Structural neuroimaging of anorexia nervosa: future directions in the quest for mechanisms underlying dynamic alterations. Biol Psychiatry 2018;83:224-34.

46. Seitz J, Herpertz-Dahlmann B, Konrad K. Brain morphological changes in adolescent and adult patients with anorexia nervosa. J Neural Transm (Vienna) 2016;123:949-59.

47. Bus BA, Molendijk ML, Penninx BJ, et al. Determinants of serum brain-derived neurotrophic factor. Psychoneuroendocrinology 2011;36: 228-39.

48. Lang UE, Hellweg R, Gallinat J. BDNF serum concentrations in healthy volunteers are associated with depression-related personality traits. Neuropsychopharmacology 2004;29:795-8.

49. Gökçe E, Güneş E, Nalçaci E. Effect of exercise on major depressive disorder and schizophrenia: a BDNF focused approach. Noro Psikiyatri Arsivi 2019;56:302-10.

50. Lim SY, Kwak Y-S. Effect of nutrients and exhaustive exercise on brain function. J Exerc Rehabil 2019;15:341-5.

51. Seifert T, Brassard P, Wissenberg $M$, et al. Endurance training enhances BDNF release from the human brain. Am J Physiol Regul Integr Comp Physiol 2010;298:R372-7.

52. Wurzelmann M, Romeika J, Sun D. Therapeutic potential of brainderived neurotrophic factor (BDNF) and a small molecular mimics of BDNF for traumatic brain injury. Neural Regen Res 2017;12:7-12.

53. Price RD, Milne SA, Sharkey J, et al. Advances in small molecules promoting neurotrophic function. Pharmacol Ther 2007;115:292-306.

54. Klein AB, Williamson R, Santini MA, et al. Blood BDNF concentrations reflect brain-tissue BDNF levels across species. Int $I$ Neuropsychopharmacol 2011;14:347-53.

55. Cao T, Li N, Cai H. Candidate metabolic biomarkers for schizophrenia in CNS and periphery: do any possible associations exist? Schizophr Res 2020;226:95-110.

56. Sohrabji F, Lewis DK. Estrogen-BDNF interactions: implications for neurodegenerative diseases. Front Neuroendocrinol 2006;27:404-14.

57. Wessels JM, Leyland NA, Agarwal SK, et al. Estrogen induced changes in uterine brain-derived neurotrophic factor and its receptors. Hum Reprod 2015;30:925-36. 
58. Cui H, Lu Y, Khan MZ, et al. Behavioral disturbances in estrogenrelated receptor alpha-null mice. Cell Rep 2015;11:344-50.

59. Young JK. Anorexia nervosa and estrogen: current status of the hypothesis. Neurosci Biobehav Rev 2010;34:1195-200.

60. Seitz J, Bühren K, Biemann R, et al. Leptin levels in patients with anorexia nervosa following day/inpatient treatment do not predict weight 1 year post-referral. Eur Child Adolesc Psychiatry 2016;25:1019-25.

61. Li C, Meng F, Lei $Y$, et al. Leptin regulates exon-specific transcription of the Bdnf gene via epigenetic modifications mediated by an AKT/p300 HAT cascade. Mol Psychiatry 2020 [Epub ahead of print]. doi:10.1038/s41380-020-00922

62. Milos G, Antel J, Kaufmann L-K, et al. Short-term metreleptin treatment of patients with anorexia nervosa: rapid onset of beneficial cognitive, emotional, and behavioral effects. Transl Psychiatry 2020; 10:303.

63. Armeanu R, Mokkonen M, Crespi B. Meta-analysis of BDNF levels in autism. Cell Mol Neurobiol 2017;37:949-54.

64. Saghazadeh A, Rezaei N. Brain-derived neurotrophic factor levels in autism: a systematic review and meta-analysis. J Autism Dev Disord 2017;47:1018-29.

65. Treasure J, Stein D, Maguire S. Has the time come for a staging model to map the course of eating disorders from high risk to severe enduring illness? An examination of the evidence. Early Intero Psychiatry 2015;9:173-84.

66. Ristori MV, Quagliariello A, Reddel S, et al. Autism, gastrointestinal symptoms and modulation of gut microbiota by nutritional interventions. Nutrients 2019;11: 2812.
67. Bandini LG, Anderson SE, Curtin C, et al. Food selectivity in children with autism spectrum disorders and typically developing children. J Pediatr 2010;157:259-64

68. Mouridsen SE, Brønnum-Hansen H, Rich B, et al. Mortality and causes of death in autism spectrum disorders: an update. Autism 2008;12:403-14.

69. Sobanski E, Marcus A, Hennighausen K, et al. Further evidence for a low body weight in male children and adolescents with Asperger's disorder. Eur Child Adolesc Psychiatry 1999;8:312-4.

70. Nakazato M, Hashimoto K, Shimizu E, et al. Decreased levels of serum brain-derived neurotrophic factor in female patients with eating disorders. Biol Psychiatry 2003;54:485-90.

71. Nakazato M, Hashimoto K, Yoshimura K, et al. No change between the serum brain-derived neurotrophic factor in female patients with anorexia nervosa before and after partial weight recovery. Prog Neuropsychopharmacol Biol Psychiatry 2006;30:1117-21.

72. Saito SKW Eri Hashimoto. Toshikazu Saito. Low serum BDNF and food intake regulation: a possible new explanation of the pathophysiology of eating disorders. Prog Neuropsychopharmacol Biol Psychiatry 2009;33:312-6.

73. Mercader JM, Ribases M, Gratacos M, et al. Altered brain-derived neurotrophic factor blood levels and gene variability are associated with anorexia and bulimia. Genes Brain Behav 2007;6: 706-16.

74. Sullivan TR, Yelland LN, Lee KJ, et al. Treatment of missing data in follow-up studies of randomised controlled trials: a systematic review of the literature. Clin Trials 2017;14:387-95. 